\title{
Analysis of Heart Rate Dynamics Before and During Meditation
}

\author{
https://doi.org/10.3991/ijoe.v17i05.11133
}

\author{
Mohammad Karimi Moridani $\left.{ }^{\bowtie}\right)$, Tina Habikazemi, Nahid Khoramabadi \\ Islamic Azad University, Tehran, Iran \\ karimi.meiautmu.ac.ir
}

\begin{abstract}
Heart rate is one of the most important vital signs. People usually face high tension in routine life, and if we found an effective method to control the heart rate, it would be very desirable. One of the goals of this paper is to examine changes in heart rate before and during meditation. Another goal is that what impact could have meditation on the human heartbeat.

To heart rate analysis before and during meditation, available heart rate signals have been used for the Physionet database that contains 10 normal subjects and 8 subjects that meditation practice has been done on them. In this paper, first is paid to extract linear and nonlinear characteristics of heart rate and then is paid to the best combination of features to identify two intervals before and during meditation using MLP and SVM classifiers with the help of sensitivity, specificity and accuracy measurements.

The achieved results in this paper showed that choosing the best combination of a feature to make a meaningful difference between two intervals before and during meditation includes two-time features (Mean HR, SDNN), a frequency feature $\left(\frac{L F}{H F}\right)$, and three nonlinear characteristics $\left(\frac{S D_{2}}{S D_{1}} \cdot S D_{2}\right.$. the maximum Lyapunov exponent). Also, using the support vector machine had better results than the MLP neural network. The sensitivity, specificity, and accuracy of the mean and standard deviation obtained respectively like $92.73 \pm 0.23,89.05 \pm 0.67,89.97 \pm 0.23$ by using MLP and respectively like $95.96 \pm 0.09,93.80 \pm 0.16$, and $94.90 \pm 0.14$ by using SVM. The results also show a valid evidence that this method can be used as a criterion of physiological impact on subjects performing meditation.

As a result, using meditation can reduce the stress and anxiety of patients by effects on heart rate, and the treatment process speeds up and have an important role in improving the performance of the system.
\end{abstract}

Keywords-Meditation; Heart rate; Signal processing; Feature extraction; Returned map

\section{$1 \quad$ Introduction}

Meditation means concentrating on the individual's mind and use to reduce stress in people and especially in patients who get depression, fear, and anxiety due to a 
particular illness [1]. Meditation can significantly help to reduce the symptoms of many diseases by making conditions of stress relief [2]. As mentioned, the purpose of using various techniques of meditation is to create and maintain a balance of health between all physical and mental aspects, and meditation is usually considered as a medical aid [3].

The present study aimed to evaluate the dynamics of heart rate signals in two different conditions. For this purpose, we used the heart rate signals of two groups of subjects (before meditation and during meditation). Another goal of this study is to find out the effect of meditation on human heart health and relaxation.

Extensive studies have been performed in the context of meditation that some of them show the effects of meditation in the medical field [4]. Dr. Herbert Benson, who works as a cardiologist at Harvard Medical School, believes that a received response from the meditation is, making more calmness in the person. His researches show that meditation's steps directly conflict and have interactions with the effects of the sympathetic nervous system (the system that makes people have more activity). While the sympathetic nervous system, dilate pupils and increase blood pressure and respiratory rate, meditation has acted oppositely and caused a reduction in blood pressure and muscle tension [5].

Meditation has many mental, psychological, and physical benefits. Therefore, it can be used in psychiatric clinics to optimize the level of stress, relaxation, attention, and so on in different people. During meditation, changes are made in biological signals that study of these changes provides valuable information about the dynamic behavior of that system [6].

This recurrence plot is a new method in processing biological signals. The main advantage of this method is to apply it for non-stationary signals. The recurrence plot is used as a nonlinear tool to analyze the heart rate signals [7]. In this method, in addition to the visualization of the status of the transition in the signal, the dimensions of the returned maps can also be used in the dynamic structure of the heart during meditation as quantitative changes.

In another study [8] to analyze the dimension of correlation and Lyapunov exponent was also shown that heart rate signals are less complicated during meditation. Also, the behavior of heart rate signal can be due to the increased parasympathetic activity and increased relaxation during meditation. In the performed study in 2008 [9] showed that an increase in the frequency peak of the heart rate signal during meditation was due to the increase of parasympathetic activity.

Because in the present study, only data from individuals with a history of meditation has been used. In the future study, also the analysis of recursive quantification can be evaluated in individuals without meditation history. This study showed that recursive quantification analysis based on complexity size can play an important role in the analysis of heart rate signals during meditation and is a convenient tool in the study of complex systems. Also, further researches can take on the classification of extractive features from the returned map to distinguish two modes before and during meditation [9].

Another study was performed with the aim of meditation effect in reduction and improvement of the symptoms of patients with heart failure in Brazil in 2005. Observing the results of this research show that meditation significantly reduced the level of 
Norepinephrine in the test group compared to the control group [10]. Also, another study was performed to estimate the depth of meditation using EEG signal and heart rate in 2011. In this research, heart rate and electroencephalogram signals before and during meditation were collected in a format of two separate data sets from 25 women. The results of this study showed that $88 \%$ of the subjects under consideration (22 of 25 ) came into the deepest level of meditation. The described algorithm in this paper has features such as the possibility to calibrate for each person and also a lack of need for high computational volume and a great time for implementing [11]. Goshvarpour and colleagues performed a study in 2012 with the aim of effecting different delays in the Poincare plot using the heart rate signals in two intervals before and during meditation. In this study, the width of the Poincare was calculated for each time delay. The analysis of achieved results showed that the width of the Poincare could be used as a criterion of changes in cardiac signals and the simplicity of plotting a Poincare plot and its matching with the chaotic nature of biological signals can be useful for evaluating of heart rate signals during meditation [12].

Other studies were performed with the aim of evaluating the effect of meditation on depression, anxiety, and stress among female students of Central and South Tehran University in 2013. The results of this study showed that the stress and anxiety of students had dropped significantly, but no differences were observed in the depression rate of control students compared to the test group [13].

Another study was performed with the aim of evaluating meditation exercises in the reduction of anxiety in patients under hemodialysis in 2015. In this study, 48 people participate that got analyzed by using descriptive statistics and statistical tests (t-test) and analysis of variance with repeated measurement. Analysis of the data showed that there is a significant difference between people who do meditation exercises and other patients [14].

The rest of the paper is structured as follows. The second section describes the database used in articles related to the heart rate of the different subjects before and during meditation, moreover suggested methods in this area. In the third section, all classification systems that have been used in MATLAB software so far are presented, and in Section IV, the summary, accuracy, and validity of all proposed methods will be presentedfrom 1998 to recent years.

\section{Materials and Methods}

\subsection{Database}

In this method, available data from the Physionet database has been used to analyze the beat's status in both cases before and during meditation that two data sets have been used [15]. Normal data (N1 to N10) are 10 healthy subjects who had no history of heart disease. Data of these people have involuntarily been recorded during sleep that the period of each of them has lasted 6 hours. To test for systematic variation across conditions the sleep period introduced 6 hours as standard by database. 
The Chi meditation data obtained in related to 5 women and 3 men who had no heart disease and in the age range between 26 to 35 years old with an average of 29 years old and cardiac signals of before and during their meditation exercises are available. Subjects have been asked to relax and do meditation exercises that each record has lasted an hour. The sampling rate of $360 \mathrm{HZ}$ and the start and end time of meditation have been specified.

\subsection{RR processing}

The RR intervals compute the duration of heartbeat cycles, which are generated by calculating the period between adjacent QRS peaks after locating the QRS complexes (see Fig.1). The QRS complexes are detected from the ECG signals using the method presented by Pan and Tompkins. Formula (1) shows how to calculate the heart rate from RR intervals [16].

$$
H R=\frac{60(\mathrm{sec})}{R R I(\mathrm{sec})}
$$

\subsection{Linear and nonlinear analysis of HRV signal}

Short term and long-term changes in heart rate have different physiological origins, and the domain of these changes can indicate a person's autonomic status. R-R intervals in the analysis of HRV are usually plotted as a function of the number of intervals and constitute a time series. Before evaluating the HRV analysis methods, there should be a clear definition of the HRV signal. In the initial definition and what comes from the HRV concept, HRV indicates the heart rate changes in the specified time periods, which are originally based on the average of R-R intervals per minute, and it has been the basis of analysis and decision making of this thesis [17].

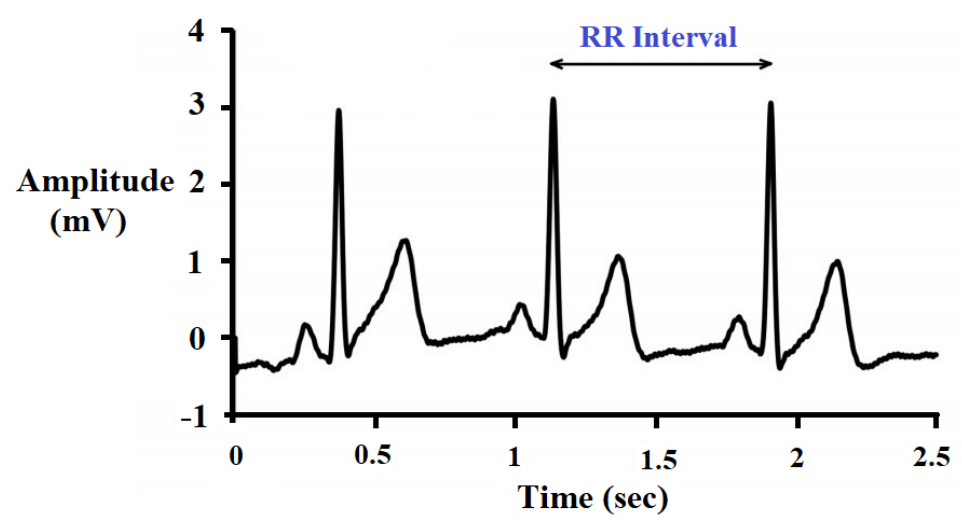

Fig. 1. Cardiac signal and display of R-R interval 
In linear methods, the total amount of changes is computed by statistical methods. Linear methods can be divided into time domain and frequency domain methods. One of the main advantages of these features is simplicity in their calculations. Of course, the statistical features somewhat depend on the quality of recorded data that this quality may be affected by environmental noises.

Features that are extracted from the time domain included a set of statistical and geometrical features such as the mean of R-R intervals (Mean HR), the standard deviation of beat-to-beat interval (SDNN), and root mean square of successive differences between normal heartbeats (RMSSD) [18].

Frequency domain methods are based on the estimation of power spectrum density that can provide useful information about how the cardiac signal is distributed as a function of frequency. The cardiac signal spectrum that is seen in healthy people usually includes harmonic components. When the power spectrum density is taken from the signal of heart rate changes, it is expected that information will be obtained about the functioning of the autonomic cardiac system that corresponds to the harmonic frequency components. Spectral analysis of HRV also can be used for short term records, even 5 minutes' pieces of the signal, and also it can be calculated for 24 hours long term records. Analysis of heart rate changes in adults has been shown that signal of heart rate changes can be divided into three frequency categories of high frequency $(0.15-0.4 \mathrm{~Hz})$, low frequency $(0.04-0.15 \mathrm{~Hz})$, and very low frequency $(0.0001-0.04 \mathrm{~Hz})$ [19]. High frequency is a parasympathetic indicator, and low frequency is an indicator of sympathetic and parasympathetic activity. The measured power spectrum density in low frequency and high-frequency bands corresponds to the autonomic balance. The increase in parasympathetic activity causes an increase in high-frequency power, while the increase in sympathetic activity causes an increase in low-frequency power. Of course, it is believed that the low-frequency band is also affected by parasympathetic activity. The physiological origin of the very low frequency is not precisely determined. The power spectrum analysis of the low frequency that has been done in 24 hours has been included frequency components of very low frequency in addition to three other frequency components. Oscillations in frequency bands of very low frequency and very low frequency are because of the mechanisms that regulate the thermal system of the body and also can be created by other factors that are still not recognized [20].

In the frequency domain is assumed that time series of R-R intervals are stationary, or in other words, changes are harmonic and sinusoidal. The fluctuations in heart rate can be periodic (because of breathing) and non-periodic (because of sudden environmental changes or individual mode). So, the signal of heart rate changes can be evaluated due to the complexity and dynamic interaction of biological signals using nonlinear methods. In the frequency domain, parameters such as VLF, HF, LF, and LF/HF can be extracted from the signal of heart rate changes [21-22].

Recent developments in the theory of nonlinear dynamics have paved a way to analyze the signals of dynamic nonlinear systems. Today recognized that nonlinear technique is able to describe processes that are caused by living biological systems. In recent years the nonlinear analysis methods have been found many applications in physiology. Changes in heart rate, blood pressure, cardiac output, blood hormone levels, and other physiological processes as obtained signals from nonlinear dynamic systems have been 
evaluated, and chaotic dynamic characteristics have been seen in their different systems.

Nonlinear analysis of the cardiac signal has been highly regarded for two main reasons. The first reason is the non-linear nature of the heart signal that acts as a nonlinear dynamic oscillator, and the second reason is the need to acquire sufficient knowledge about this real phenomenon [23].

Many researchers have been very interested in the analysis of heart rate signals because this signal has much information in itself, which is directly concerned with heart health [24] and heart diseases such as heart failure [25], myocardial infarction [26] and angina [27]. In addition, this signal is a valuable non-invasive tool for describing the role of the autonomous nervous system in the regulation of the circulatory system. Initial analysis of time-series was based on a statistical index that this analysis gave way to a more sophisticated analysis very soon to extract valuable information from the signal.

Because of the chaotic behavior of the cardiovascular system, nonlinear methods used in the analysis of the heart rate signal. One of these techniques is the Poincare plot. This method was used as a qualitative tool for the first time, and a little later, the geometry quantification of the Poincare plot proposed. Tulppo et al. [28] placed an oval on the Poincare plot to calculate the index of heart rate. Brennan et al. [29] showed that the Poincare plot width shows the level of short-term changes in the heart rate signal.

Poincare plot is a relatively new method for analyzing nonlinear dynamical system such as the HRV signal. Each point determined on this graph as (RR (n), RR $(n+1)) n$ $=1,2,3 \ldots \mathrm{k}$ that $\mathrm{k}$ is the signal length [30]. Regarding statistical, this chart graphically shows the correlation between R-R successive intervals, but its original and important concept is that this graph is a two-dimensional state space of successive intervals, which represents its nonlinear dynamics. Also, this graph gives useful information about the oscillations of the short and long term. Poincare plot analyzed quantitatively by calculating the standard deviation of $R R(n)$ intervals with the $y=x$ and $y=-x+2 R R m$ lines, which the RRm is the RR (n) s mean. Standard deviations called SD1 and SD2, which SD1 is related to the rapid changes of the heartbeat to BPM data that is mainly related to respiratory sinus arrhythmia. While SD2 describes the long-term changes in RR (i)s. Also, the SD2/ SD1 ratio can be calculated to describe the relationship between these components [31]. The SD1 and SD2 amounts of Poincare plot directly depend on the statistical amounts of the standard deviation of the heart rate signal and standard deviation of two consecutive distances of $\mathrm{R}$ peaks and are calculated by the formula (2). For the formation of heart rate variability of time series, the opposite of the heart rate values is used according to formula (2). Figure 2 shows a cardiac signal before and during meditation with the Poincare plot.Table 1 shows linear and non-linear extracted features from the HRV signal.

$$
\begin{array}{r}
S D_{1}=\frac{\sqrt{2}}{2} S D\left(R R_{i+1}-R R_{i}\right) \\
S D_{2}=\sqrt{2 S D\left(R R_{i}\right)^{2}-\frac{1}{2} S D\left(R R_{i+1}-R R_{i}\right)^{2}}
\end{array}
$$


Table 1. Linear and non-linear extracted features from HRV signal

\begin{tabular}{|c|c|c|c|}
\hline Variable & Units & Description & Equation \\
\hline Mean HR & $\mathrm{ms}$ & $\begin{array}{l}\text { Mean of RR intervals (RRIs) } \\
\text { (Mean HR) }\end{array}$ & $\begin{array}{l}\text { Mean } H R=\frac{\sum_{i=1}^{N} N N(i)}{N} \\
\text { where } N \text { is the total number of all RRIs in a } \\
\text { given segment }\end{array}$ \\
\hline SDNN & $\mathrm{ms}$ & $\begin{array}{l}\text { The standard deviation of all } \\
\text { RRIs (SDNN) }\end{array}$ & $S D N N=\sqrt{\frac{1}{N}\left(\sum(R R(i)-\text { Mean } H R)^{2}\right)}$ \\
\hline SDANN & $\mathrm{ms}$ & $\begin{array}{l}\text { The standard deviation of the av- } \\
\text { erage RRIs in all 1-minute sec- } \\
\text { tion which they divide selected } \\
\text { segments of long-term signal } \\
\text { (SDANN) }\end{array}$ & $\begin{array}{l}\text { SDANN }=\sqrt{\frac{1}{N}}\left(\sum_{i=1}^{N}\left(\text { Mean }_{i}-\text { Mean }_{\text {all }}\right)^{2}\right. \\
\text { where } N \text { is the total number of } 1-\text { minute sec- } \\
\text { tions RRIs in the selected segment, } \text { Mean }_{i} \text { is } \\
\text { mean of RR interval in } 1 \text { minutes section, } \\
\text { Mean }_{\text {all }} \text { is mean of all } 1 \text { minutes sections. }\end{array}$ \\
\hline RMSSD & $\mathrm{ms}$ & $\begin{array}{l}\text { Root mean square of successive } \\
\text { differences between adjacent } \\
\text { RRIs (RMSSD) }\end{array}$ & $R M S S D=\sqrt[2]{\frac{1}{N}}\left(\sum_{i=1}^{N}\left(R R_{i+1}-R R_{i}\right)^{2}\right.$ \\
\hline LF & $\mathrm{ms}^{2}$ & $\begin{array}{l}\text { The absolute power of the low- } \\
\text { frequency band (0.04-0.15), an } \\
\text { estimate of long-term HRV. }\end{array}$ & \\
\hline $\mathrm{HF}$ & $\mathrm{ms}^{2}$ & $\begin{array}{l}\text { The absolute power of the high- } \\
\text { frequency band }(0.15-0.4) \text {, an } \\
\text { estimate of the short term of } \\
\text { HRV. }\end{array}$ & \\
\hline$\frac{L F}{\mathrm{HF}}$ & $\%$ & The ratio of LF to HF power & \\
\hline $\mathrm{SD}_{1}$ & ms & $\begin{array}{l}\text { Poincare plot standard deviation } \\
1 \text { (SD1), associated with para- } \\
\text { sympathetic activity }\end{array}$ & $\frac{\sqrt{2}}{2} S D\left(R R_{i+1}-R R_{i}\right)$ \\
\hline $\mathrm{SD}_{2}$ & $\mathrm{~ms}$ & $\begin{array}{l}\text { Poincare plot standard deviation } \\
2 \text { (SD2), associated with sympa- } \\
\text { thetic activity. }\end{array}$ & $\sqrt{2 S D\left(R R_{i}\right)^{2}-\frac{1}{2} S D\left(R R_{i+1}-R R_{i}\right)^{2}}$ \\
\hline$\frac{S D 2}{\text { SD1 }}$ & $\%$ & $\begin{array}{l}\text { The ratio of SD2 to SD1, de- } \\
\text { scribe the relationship between } \\
\text { sympathetic to parasympathetic } \\
\text { activity }\end{array}$ & \\
\hline $\begin{array}{l}\text { Maximal Lya- } \\
\text { punov exponent }\end{array}$ & & $\begin{array}{l}\text { The average rate of divergence } \\
\text { of two neighboring trajectories }\end{array}$ & $\begin{array}{l}\quad \lambda=\lim _{N \rightarrow \infty} \frac{1}{N} \sum_{n=1}^{N} \ln \left(\frac{d x_{n+1}}{d x_{n}}\right) \\
\mathrm{x}_{\mathrm{n}} \text { is a time series, and } \mathrm{N} \text { is the number of } \\
\text { samples } \\
\text { Two close points at step } \mathrm{n}, \mathrm{x}, \text { and } \mathrm{x}_{\mathrm{n}}+\mathrm{dx}_{\mathrm{n}}\end{array}$ \\
\hline Entropy & & $\begin{array}{l}\text { Entropy measures the regularity } \\
\text { and complexity of a time series }\end{array}$ & $\begin{array}{l}\qquad E(\alpha)=\frac{1}{1-\alpha} \log _{2}\left(\sum_{i=1}^{n} p_{i}^{\alpha}\right) \\
\text { Where } p_{i} \text { is the probability that a random var- }- \\
\text { iable takes a given value out of } n \text { values, and } \\
\alpha \text { is the order of the entropy measure. }\end{array}$ \\
\hline
\end{tabular}




\subsection{Feature extraction}

In diagnostic and forecasting systems for choosing the best combination of features is very important to make the most distinction between the groups. So first performed each feature separately classified according to the highest sensitivity and specificity, the best features are selected. Then combine this feature with every feature; the best binary composition will be obtained. Similarly, this process continues until the best resulting combination that makes the greatest difference between the groups is achieved[17].

The selection of a large number of feature functions makes the classifier confused and leads to its failure to distinguish between the two groups of features extracted from the two categories of the signal [32]. Since some of the features are incapable of proper classification, the feature dimensions are reduced with the principal component analysis (PCA) method. The selection of a few features prevents highlighting the properties and state of a signal and fails to distinguish between two different signals (before and during meditation).

In this paper, from the features listed in Table 1, the best combination has 6 features that are two-time features (Mean HR, SDNN), a frequency feature $\left(\frac{L F}{H F}\right)$, and three nonlinear characteristics $\left(\frac{S D_{2}}{S D_{1}} \cdot S D_{2}\right.$, the maximum lyapunov exponent).
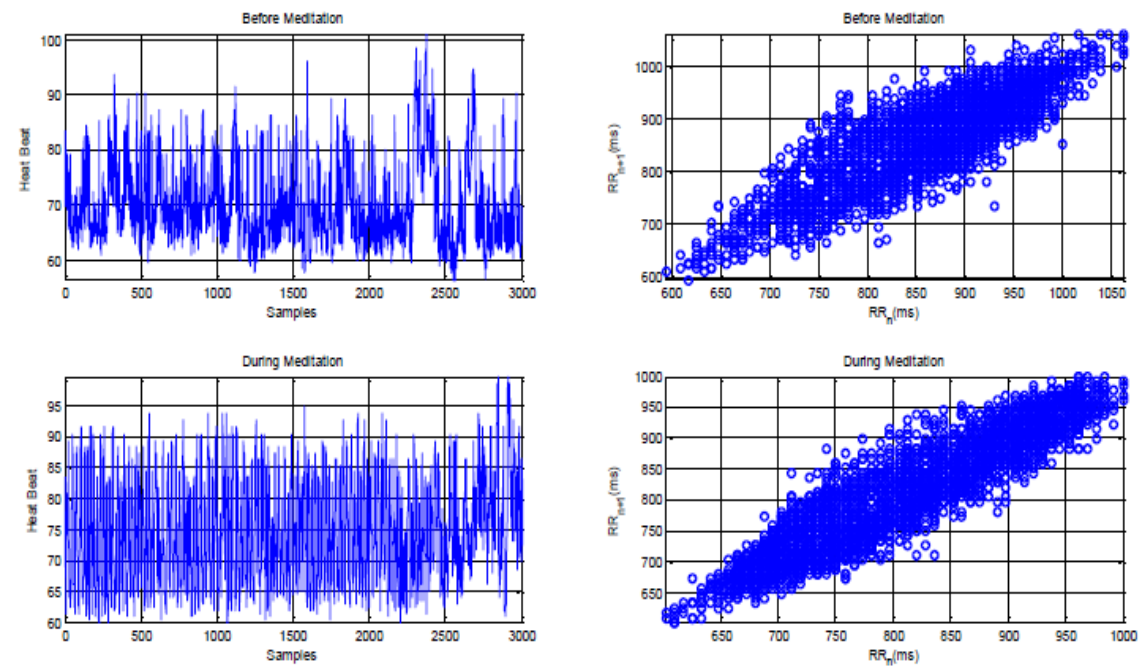

Fig. 2. Display of cardiac signal before and during meditation (left), along with Poincare plot (right)

\subsection{Diagnostic system using neural networks}

The most common way to classify is the use of artificial neural networks. An artificial neural network is an information processing system, which its executive features 
are similar and common with the performance of live neural networks in humans. In the following two successful methods that are used in the classification of biological signals, are introduced.

\subsection{Multi-layer perceptron neural network}

The multi-layer perceptron neural network, usually used in the classification issues that is formed of an input layer, one or more hidden layers, and an output layer. In this structure, all of the neurons in one layer are connected to all of the neurons in the next layer, and in fact; they form a network with full connections. In this network, the number of neurons in each layer is independent of the number of other layer neurons [3334].

To use an appropriate classification using the supervised neural network, the first step is to choose the network dimensions. The input dimension determines the dimension of the input layer, and also, in the output layer, neurons should be laid to the number of classes. Ideally, with a corresponding entry to any class or group, expect that the related neuron of that class takes one value, and other neurons take zero value on their own. Due to using the sigmoid function, the network output value will be a number between zero and one. Thus, in practice, the neuron of the output layer that its value is much larger than the other neurons will specify the class of that data.

In the case of neurons in the middle layer, there is no special basis and usually are chosen by trial-and-error method so that the network will have a reasonable answer. It should be noted in this issue that if the network is very complex, will learn the behavior of the input pattern exactly, and if the data change slightly than the training data, the network will not easily be able to pursue it [35].

The MLP network has a two-layer feed-forward that uses an error propagation algorithm that will be trained with a variable learning rate. The number of neurons of the first layer equal to the number of features considered, and the output layer contains a neuron that has values between zero and one that related to the two groups before and during the meditation. Also, the activity function of neurons selected of the tangent sigmoid to change the number of neurons in the middle layer is attempting to optimize the neural network architecture [36]. Some common structures types of the artificial neural network are reviewed, and the best-resulted network is a two-layer neural network with 5 neurons in the hidden layer with the standard sigmoid activity function. Network training will be continued until the square of error is less than 0.01 , or the number of repetitions of training will be 1000 . Since the number of data used is limited, this network according to the Leave One Out method in each level one of the observations as test data, and the rest are selected as training data and in the next level the second data as test data and the rest are selected as training data, and this action will be continued until all data participate in the test and training phase [37]. Finally, network error extracted, and the average is calculated. One advantage of this method is that all data participate in the training and testing level and are causing an increase in network performance. 


\subsection{Support vector machine}

A support vector machine is one of the learning methods to monitor that is used for classification, clustering, and modeling [38]. This method has shown good performance compared to other methods of categorizing, including perceptron neural networks. The basis of support vector machine work is linear categories of data, and in the linear dividing of data tries to choose a line that has a greater safety margin. In this method, by using an optimization algorithm, the samples which form the class borders will be achieved, which call backup vectors. Teaching points that are closest to the point of decision-making can be considered as a subset to define boundaries for decision-making and to be a support vector [39].

The advantages of support vector machine are its relatively simple training, and for data with high dimension have almost good answers. Support vector machine, unlike types of neural networks instead of minimizing error, tries to minimize the operational risk by classifying or modeling. The main difference of support vector machine to neural networks is in some neural networks if the error rate of the whole network is less than a certain threshold, they stop learning network, but support vector machine looking for the optimal solution. In other words, a compromise between the complexity of the categorization and the error rate is controlled. Also, other neural networks, such as MLP, may be stopped at the national maximum. But the mode of support vector machine operation is not the case. For better separation of data in SVM, data are mapped to higher space by a nonlinear function, because separation is done easier in higher space [40].

SVM is a nonlinear classifier that uses kernel functions to map the features on higher dimensional space; to obtain a linear optimal hyperplane. The obtained hyperplane is capable of separating features before and during meditation. The selection of the proper kernel function has a significant impact in distinguishing the two groups. In this paper, various kernel functions, including linear, quadratic, polynomial, and radial basis function $(\mathrm{RBF})$ are used to map features on higher dimensional space. The degree of polynomial kernel function is changed from 1 to 5 , and the scaling factor of RBF is changed from 0.5 to 1.5 with 0.1 steps.

\subsection{Classifier performance evaluation}

There are several methods to judge the performance of different classifiers, and various parameters can be considered for this purpose that many of the most common of these are accuracy, sensitivity, and specificity. The Accuracy of the test is as formula (3).

$$
\operatorname{Accuracy}(\%)=\frac{(T P+T N)}{(T P+F N+T N+F P)} \times 100
$$

Which TP, TN, FP, FN are True Positive, True Negative, False Positive, False Negative, respectively.

The sensitivity of the test is its ability to identify the true positive or correctly identify those who are sick. For example, when told that a test sensitivity is $80 \%$ it means that 
the desired test can detect $80 \%$ of sickness, and in other words, such this test will result in $80 \%$ of cases is true-positive, and in $20 \%$ of cases, false-negative and so $20 \%$ of patients cannot be distinguished. The sensitivity formula is shown in formula (4).

$$
\text { Sensitivity }(\%)=\frac{T P}{T P+F N} \times 100
$$

The specificity of the test is its ability to identify the true negative or correctly identify those who are not really sick. For example, when told that a test specificity is $70 \%$, it means that the desired test can detect $70 \%$ of healthy people. In other words, such this test will result in $70 \%$ of cases is a true negative, and 30\% of cases are false-positive and so $30 \%$ of healthy people introduced as a patient. The specificity of a test is the ratio of true negatives. The specificity formula is shown in formula (5).

$$
\text { Specificity }(\%)=\frac{T N}{T N+F P} \times 100
$$

The sensitivity and specificity of a test indicate that the test is positive or not in healthy and patient's subjects and, therefore, can be used as a diagnostic tool. They are the most suitable tests with high sensitivity and accuracy, but it is difficult to make a balance between these two parameters and is highly dependent on the type of selected features [18].

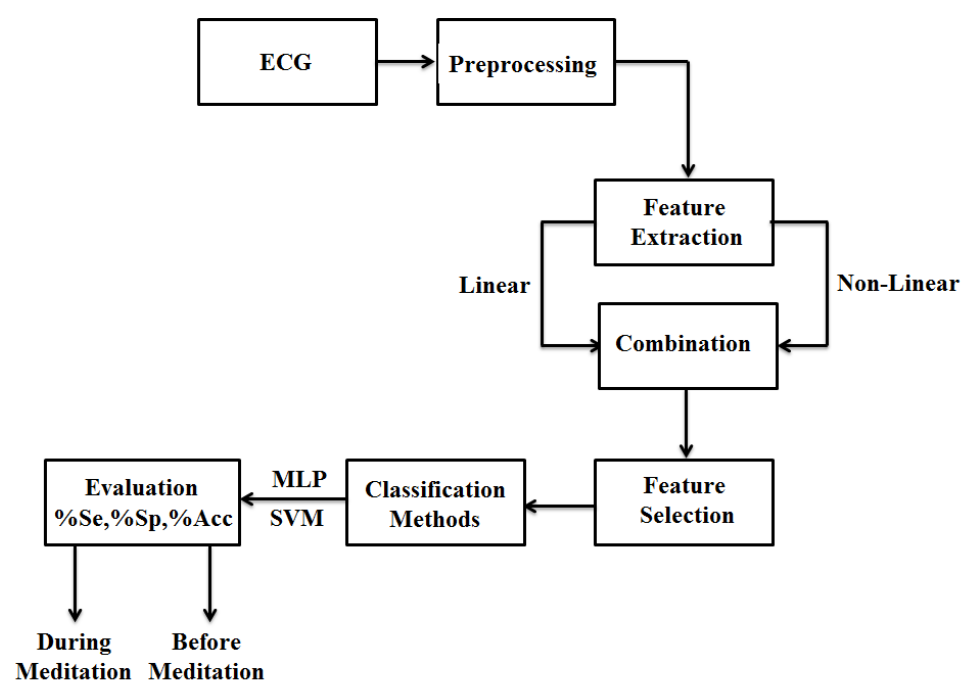

Fig. 3. Diagnosis steps before and during meditation

The distribution of feature space of heart rate is shown in Figure 4. As seen in this Figure, this feature can provide an appropriate resolution between the two intervals before and during meditation. 


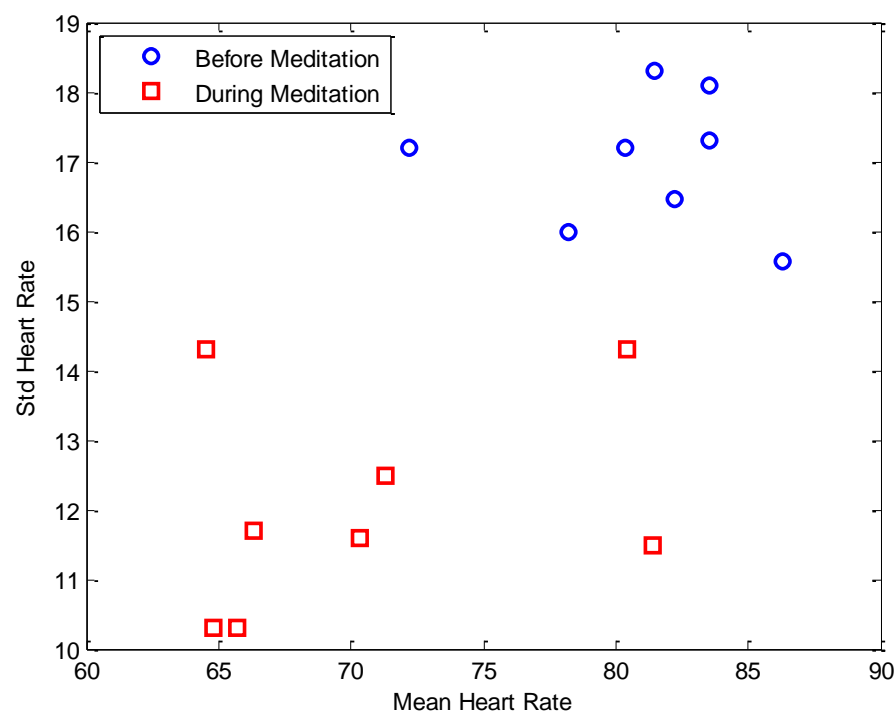

Fig. 4. Distribution of feature space of heart rate

\subsection{Leave-One-Out Cross Validation}

Leave-One-Out Cross Validation (LOOCV) is a special case of K-fold cross validation with $K=n$, where $\mathrm{n}$ is the total number of samples in the training multiset. $n$ experiments are performed using $n-1$ samples for training and the remaining sample for testing. If the original sample is small, a larger $K$ may be better. In this study, the number of samples (n) is 8 . This method is usually used in cases where it is difficult to obtain labeled data. It is rather computationally expensive, if the number of data is large [41].

\section{Simulation Results}

After extraction of various linear and nonlinear characteristics of the heart rate signal in two intervals before and during meditation, the combination of features and finally select the best combination of features will be discussed. Finally, the best combination of features was used as input for an artificial neural network and support vector machine. Table 2 shows the mean values and standard deviation of linear characteristics, and Table 3 shows the values listed for nonlinear characteristics in two intervals before and during the meditation. Figure 5 shows the comparison of the changes in the best combination of the selected feature in two intervals before and during the meditation. 
Table 2. Mean values and SD of linear characteristics before and during the meditation

\begin{tabular}{|l|c|c|c|c|c|c|c|}
\hline $\begin{array}{c}\text { Time duration } \\
\text { Classic Features }\end{array}$ & Mean HR & SDNN & SDANN & RMSSD & LF & HF & $\frac{\boldsymbol{L F}}{\boldsymbol{H F}}$ \\
\hline Before meditation & $80.35 \pm 17.2$ & $105 \pm 27$ & $3.95 \pm 5.6$ & $38.3 \pm 13.7$ & $344 \pm 169$ & $258 \pm 40$ & $1.33 \pm 1.12$ \\
\hline During meditation & $66.32 \pm 11.69$ & $95 \pm 22.7$ & $2.24 \pm 4.8$ & $29.6 \pm 10.18$ & $390 \pm 143$ & $228 \pm 28$ & $1.71 \pm 1.25$ \\
\hline P-value & 0.046 & 0.063 & 0.052 & 0.043 & 0.072 & 0.061 & 0.057 \\
\hline
\end{tabular}

Table 3. Mean values and SD of nonlinear characteristics before and during the meditation

\begin{tabular}{|l|c|c|c|c|c|}
\hline $\begin{array}{c}\text { Time duration Classic } \\
\text { Features }\end{array}$ & SD1 & SD2 & $\frac{\boldsymbol{S D}_{\mathbf{2}}}{\boldsymbol{S D}_{\mathbf{1}}}$ & $\begin{array}{c}\text { Maximum } \\
\text { Lyapunov } \\
\text { Exponent }\end{array}$ & Entropy \\
\hline Before meditation & $7.49 \pm 3.52$ & $34.18 \pm 16.43$ & $4.98 \pm 0.04$ & $0.46 \pm 0.08$ & $4.44 \pm 0.28$ \\
\hline During meditation & $6.87 \pm 3.11$ & $45.79 \pm 18.40$ & $6.11 \pm 0.05$ & $0.31 \pm 0.04$ & $3.37 \pm 0.2$ \\
\hline P-value & 0.042 & 0.043 & 0.039 & 0.013 & 0.028 \\
\hline
\end{tabular}
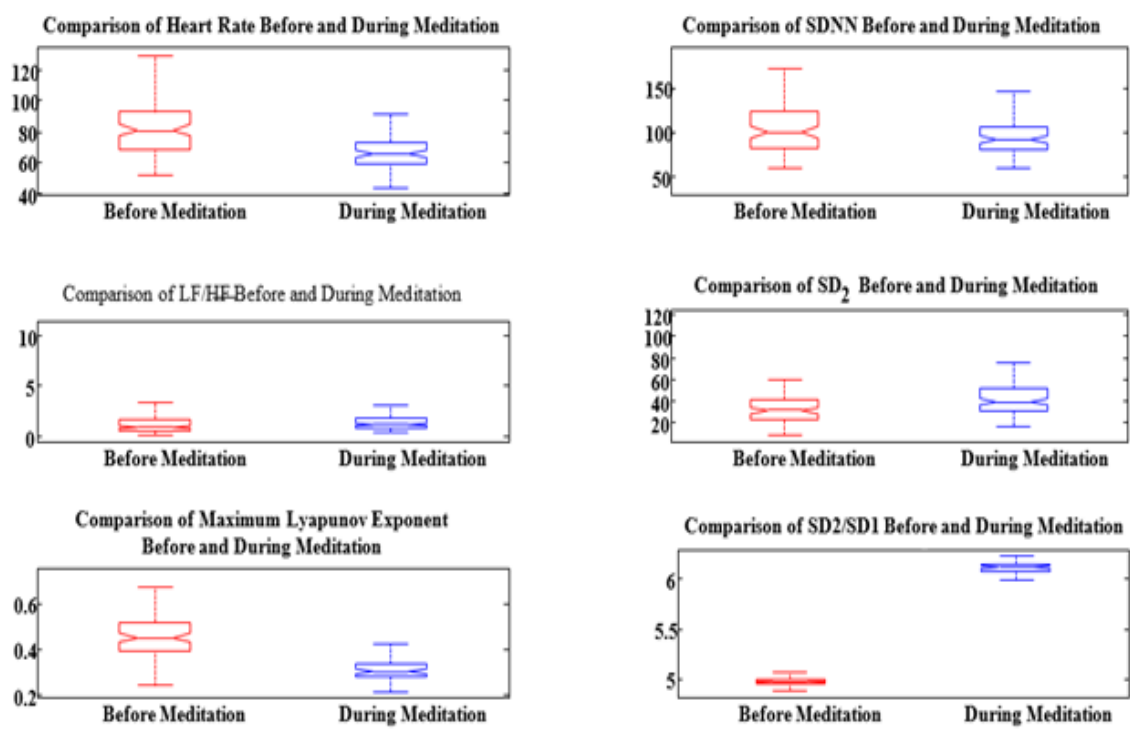

Fig. 5. Comparison of the changes inmean and variance values of the best combination of the selected feature

The results of the sensitivity, specificity, and accuracy of the system proposed in this paper use MLP and SVM neural network to determine the period before and during meditation to help select the best combination of features are shown in Table 4. These results are based on test data. 
Table 4. Evaluation results of MLP and SVM classifier system

\begin{tabular}{|l|c|c|c|c|c|c|}
\hline \multirow{3}{*}{ Classifier } & \multicolumn{3}{|c|}{ MLP } & \multicolumn{3}{c|}{ SVM } \\
\cline { 2 - 7 } & $\begin{array}{c}\text { Sensitivity } \\
(\boldsymbol{\%})\end{array}$ & $\begin{array}{c}\text { Specificity } \\
(\boldsymbol{\%})\end{array}$ & $\begin{array}{c}\text { Accuracy } \\
(\boldsymbol{\%})\end{array}$ & $\begin{array}{c}\text { Sensitivity } \\
(\boldsymbol{\%})\end{array}$ & $\begin{array}{c}\text { Specificity } \\
(\boldsymbol{\%})\end{array}$ & $\begin{array}{c}\text { Accuracy } \\
(\boldsymbol{\%})\end{array}$ \\
\hline Before Meditation & $92.25 \pm 0.24$ & $88.34 \pm 0.72$ & $89.12 \pm 0.38$ & $95.76 \pm 0.08$ & $93.18 \pm 0.17$ & $94.37 \pm 0.16$ \\
\hline During Meditation & $93.21 \pm 0.22$ & $89.76 \pm 0.62$ & $90.81 \pm 0.32$ & $96.16 \pm 0.10$ & $94.42 \pm 0.15$ & $95.42 \pm 0.12$ \\
\hline Average & $92.73 \pm 0.23$ & $89.05 \pm 0.67$ & $89.97 \pm 0.23$ & $95.96 \pm 0.09$ & $93.80 \pm 0.16$ & $94.90 \pm 0.14$ \\
\hline
\end{tabular}

\section{Discussion}

Meditation is typically considered as a medical aid method. The goal of meditation techniques is to make and maintain a balance of health between all physical and mental aspects [42]. In recent years, extensive researches have focused on controlling some mental and physical illnesses using meditation and its effects on the mind [43-46]. Also, meditation is considered a method in managing psychological diseases (such as anxiety and depression) and maintaining the health of the mind [47-48]. Researches have shown that physiologically, meditation causes a reduction in metabolic activity, increases the coalition and order in brain function, reduces the peripheral vascular resistance, and increases the blood flow of the brain [48]. Also, it is different from sleep or simple relaxation. This technique has improvements in mental health and increases positive features and reduces psychological anxiety [49]. According to the great benefits of meditation, scientific studies are important in this phenomenon.

The heart rate signal is a valuable non-invasive tool for describing the role of the autonomic nervous system in regulating the circulatory system. The first analyses of the time series were based on the statistical indexes that these analyses replaced with more complex analyzes very soon to extract valuable information from the signal [7, 50]. Initial analyses, such as spectral analysis, have limiting assumptions for time series of heart rate and are conditioned with many changes of noise sources. In these methods, the signal is considered static, and tests are carried out under controlled circumstances. To provide a solution for the non-stationary signal issue, the method of analyzing nonlinear dynamics and chaos theory is used to quantify these dynamics. One of these techniques is the analysis of the returned map.

Researches have shown that physiologically, meditation causes a reduction in metabolic activity, increases the coalition and order in brain function, reduces the peripheral vascular resistance, and increases the blood flow of the brain. Meditation is different from sleep or simple relaxation. This technique has improvements in mental health and increases positive features and reduces psychological anxiety. According to the great benefits of meditation, scientific studies are important in this phenomenon.

In most meditation techniques, the individual focuses on breathing, and since breathing oscillations are modeled on the heart rate signal, most studies have been performed on the heart rate signal. Due to the interaction of biological systems with each other, changes occur in all vital signals, including electroencephalogram signals during meditation. The study of these changes provides useful information about brain function. 


\section{Conclusion}

Meditation can reduce fatigue in the minds of athletes. This is one of the subjects of sports medicine. High sports activity may cause muscle tension, anxiety, and disorder in focus. Researches have shown that meditation increases concentration and the control of heart rate.

Because in the present study, only data from individuals with a history of meditation has been used. In the future study, also the analysis of recursive quantification can be evaluated in individuals without meditation history. This study showed that recursive quantification analysis based on complexity size can play an important role in the analysis of heart rate signals during meditation and is a convenient tool in the study of complex systems. Also, further researches can take on the classification of extractive features from the returned map to distinguish two modes before and during meditation. The results showed that meditation is effective in reducing heart rate and calm the people. Since meditation is a simple and low-cost method and considering that reduction and increased heart rate can be very problematic and dangerous for the patient, one of the most common problems of patients can be reduced by the implementation of the meditation exercises.

\section{Abbreviations}

ECG: Electrocardiogram

EEG: Electroencephalogram

FN: False Negative

FP: False Positive

HF: High frequency

HR: Heart Rate

HRV: Heart Rate Variability

MLP: Multilayer perception

LF: Low frequency

PCA: Principal component analysis

RBF: Radial basis function

RMSSD: Root mean square of successive differences

SDNN: Standard deviation of beat-to-beat interval

SVM: Support vector machine

TN: True Negative

TP: True Positive

VLF: Very low frequency 


\section{$7 \quad$ References}

[1] Hofmann, S. G., Sawyer, A. T., Witt, A. A., \& Oh, D. (2010). The effect of mindfulnessbased therapy on anxiety and depression: A meta-analytic review. Journal of consulting and clinical psychology, 78(2), 169-183. https://doi.org/10.1037/a0018555

[2] Vandana, Balakrishnan et al. "Meditation induces a positive response during stress events in young Indian adults." International journal of yoga vol. 4, 2 (2011): 64-70. https://doi.org/10.4103/0973-6131.85487

[3] Javanbakht M, Kenari RH, Ghasemi M. Effects of yoga on depression and anxiety of women. Complement Ther Clin Pract. 2009; 15: 102-4. https://doi.org/10. 1016/j.ctcp.2009.01.003

[4] Murphy M, Donovan S, Taylor E. The physical and physiological effects of meditation: a review of contemporary research with a comprehensive bibliography 1931-1996. Sausalito, CA: Institute Noeti Science. 1997: 1-23.

[5] Benson Herbert. 1974. Transcendental Meditation - Science or Cult? Journal of the American Medical Association 227:807.

[6] Curiati JA, Bocchi E, O FJ, et al. Meditation reduces sympathetic activation and improves the quality of life in elderly patients with optimally treated heart failure. J Altern Complement Med. 2005; 11(3): 465-7. https://doi.org/10.1089/acm.2005. 11.465

[7] Ateke Goshvarpour, Atefeh Goshvarpour. Comparison of higher order spectra in heart rate signals during two techniques of meditation: Chi and Kundalini meditation, Cogn Neurodyn. 2013 Feb; 7(1): 39-46. https://doi.org/10.1007/s11571-012-9215-z

[8] Marwan, N., Kurths, J.: Cross Recurrence Plots and Their Applications, in: Mathematical Physics Research at the Cutting Edge, Hauppauge, Nova Science Publishers, 2004, 101-139.

[9] Raghavendra B. S., Dutt D. N. Multiscale fractal dimension technique for characterization and analysis of biomedical signals. Proceedings of the Digital Signal Processing and Signal Processing Education Meeting (DSP/SPE '11); January 2011; Sedona, Ariz, USA. IEEE; pp. 370-374. https://doi.org/10.1109/dsp-spe.2011.5739242

[10] Phongsuphap S., Pongsupap Y., Chandanamattha P., Lursinsap C. (2008). Changes in heart rate variability during concentration meditation. Int. J. Cardiol. 130, 481-484. https://doi.org/10.1016/j.ijcard.2007.06.103

[11] A Goshvarpour, A Goshvarpour, Chaotic Behavior of Heart Rate Signals during Chi and Kundalini Meditation, International Journal of Image Graphics and Signal Processing, ZUMS Journal,2012, 4 (2), 23-29. https://doi.org/10.5815/ijigsp.2012.02.04

[12] Dehghani S, Amini K, Shakibazadeh E, Faghih Zadeh S، Hashem Zadeh M5, Effects of Two Heart Meditation Exercise on Anxiety among Patients Undergoing Hemodialysis, Preventive Care in Nursing \& Midwifery Journal (PCNM),2015, Vol. 4, No.2, 56-65.

[13] Karimi A, Bahreinian SA, Ghobaribonab B. An investigation of the impact of meditation on anxiety and depression of female students. J of Clinic Psych Stud. 2013; 9(3): 99-115 [In Persian].

[14] Dharmawardene M, Givens J, Wachholtz A, Makowski S, Tjia J. A systematic review and meta-analysis of meditative interventions for informal caregivers and health professionals. Br Med J 2015; 6: 160-169. https://doi.org/10.1136/bmjspcare-2014-000819

[15] Exaggerated Heart Rate Oscillations during Two Meditation Techniques: https://www.physionet.org/physiobank/database/meditation/data/.Last accessed: 16-2-2-17.

[16] F. Marzbanrad et al., The effect of automated preprocessing of RR interval tachogram on discrimination capability of heart rate variability parameters, computing in cardiology, 2013, 483-486. 
[17] Soroor Behbahani, Mohammad Karimi Moridani, Non-linear Poincaré analysis of respiratory efforts in sleep apnea, Bratislavske lekarske listy, 116(7), 2015, 426-432. https://doi.org/10.4149/bll_2015_081

[18] Mohammad Karimi Moridania, Seyed Kamaledin Setarehdanb, Ali Motie Nasrabadic and Esmaeil Hajinasrollah, Non-linear feature extraction from HRV signal for mortality prediction of ICU cardiovascular patient, Journal of Medical Engineering \& Technology, 2016, 40(3), 87-98. https://doi.org/10.3109/03091902.2016. 1139201

[19] Berntson GG, Bigger JT Jr, Eckberg DL, Grossman P, Kaufmann PG, Malik M, Nagaraja HN, Porges SW, Saul JP, Stone PH, van der Molen MW, Heart rate variability: origins, methods, and interpretive caveats, Psychophysiology. 1997 Nov; 34(6):623-48. https://doi.org/10.1111/j.1469-8986.1997.tb02140.x

[20] Novak V, Saul JP, Eckberg DL. Task Force report on heart rate variability, Circulation. 1997 Aug 5; 96(3):1056-7.

[21] Pagani M, Lombardi F, Guzzetti S, Rimoldi O, Furlan R, Pizzinelli P, Sandrone G, Malfatto G, Dell'Orto S, Piccaluga E, et al. Power spectral analysis of heart rate and arterial pressure variabilities as a marker of sympatho-vagal interaction in man and conscious dog, Circ Res. 1986 Aug;59(2):178-93. https://doi.org/10.1161/01.res.59.2.178

[22] Malliani A, Pagani M, Lombardi F, Cerutti S. Cardiovascular neural regulation explored in the frequency domain, Circulation. 1991 Aug; 84(2):482-92.https://doi.org/10.1161/01.cir. $\underline{84.2 .482}$

[23] I. Radojicic, D. Mandic and D. Vulic, on the presence of deterministic chaos in HRV signals, Computers in Cardiology2001. Vol.28, Rotterdam, Netherlands, 2001, pp. 465-468, https://doi.org/10.1109/cic.2001.977693

[24] Peng CK, Mietus JE, Liu Y, Khalsa G, Douglas PS, Benson H, Goldberger AL. Exaggerated heart rate oscillations during two meditation techniques. Int J Cardiol. 1999 Jul 31; 70(2):101-7. https://doi.org/10.1016/s0167-5273(99)00066-2

[25] Nolan J1, Batin PD, Andrews R, Lindsay SJ, Brooksby P, Mullen M, Baig W, Flapan [AD, Cowley A, Prescott RJ, Neilson JM, Fox KA. Prospective study of heart rate variability and mortality in chronic heart failure: results of the United Kingdom heart failure evaluation and assessment of risk trial (UK-heart), Circulation. 1998 Oct 13; 98(15):1510-6. https://doi.org/10.1161/01.cir.98.15.1510

[26] Kleiger RE, Miller JP, Bigger JT Jr, Moss AJ. Decreased heart rate variability and its association with increased mortality after acute myocardial infarction, Am J Cardiol. 1987 Feb 1; 59(4):256-62. https://doi.org/10.1016/0002-9149(87)90795-8

[27] G A Lanza, D Cianflone, A G Rebuzzi, G Angeloni, A Sestito, G Ciriello, G La Torre, F Crea, and A Maseri, Prognostic value of ventricular arrhythmias and heart rate variability in patients with unstable angina, Heart. 2006 Aug; 92(8): 1055-1063.https://doi.org/10. 1136/hrt.2005.070714

[28] Tulppo MP, Mäkikallio TH, Takala TE, Seppänen T, Huikuri HV. Quantitative beat-to-beat analysis of heart rate dynamics during exercise, Am J Physiol. 1996 Jul; 271(1 Pt 2):H24452. https://doi.org/10.1152/ajpheart.1996.271.1.h244

[29] Brennan MJ, Delogu G, Chen Y, Bardarov S, Kriakov J, Alavi M, Jacobs WR Jr. Evidence that mycobacterial PE_PGRS proteins are cell surface constituents that influence interactions with other cells, Infect Immun. 2001 Dec;69(12):7326-33.https://doi.org/10.1128/ iai.69.12.7326-7333.2001

[30] Lerma C, Infante O, Pérez-Grovas H, José MV. Poincaré plot indexes of heart rate variability capture dynamic adaptations after haemodialysis in chronic renal failure patients, Clin Physiol Funct Imaging. 2003 Mar; 23(2):72-80. https://doi.org/10. $\underline{1046 / j .1475-097 x .2003 .00466 . x}$ 
[31] Agnieszka Kitlas Golińska1. Poincar'e Plots in Analysis of Selected Biomedical Signals, Studies in Logic, Grammar and Rhetoric, 2013, 35 (48), 117-127. https://doi.org/10. 2478/slgr-2013-0031

[32] MK Moridani, SK Setarehdan, AM Nasrabadi, E Hajinasrollah, Analysis of heart rate variability as a predictor of mortality in cardiovascular patients of intensive care unit, Biocybernetics and Biomedical Engineering 35 (4), 217-226,2015.https://doi.org/10.1016/ j.bbe.2015.05.004

[33] Lewicke A, Sazonov E, Corwin MJ, Neuman M, Schuckers S; CHIME Study Group. Sleep Versus Wake Classification from Heart Rate Variability Using Computational Intelligence: Consideration of Rejection in Classification Models, IEEE Trans Biomed Eng. 2008, 55 (1), 108-118. https://doi.org/10.1109/tbme.2007.900558

[34] F. Yaghouby, A. Ayatollahi, R. Soleimani, Classification of cardiac abnormalities using reduced features of heart rate variability signal, World Applied Sciences Journal, 6 (2009), pp. $1547-1554$.

[35] Mohammad Karimi Moridani, Shahrzad Marjani, A Review of the Methods for Sudden Cardiac Death Detection: A Guide for Emergency, International Journal of Online and Biomedical Engineering (iJOE), 2020,16(9),137-158.https://doi.org/10.3991/ijoe.v16i09. $\underline{14485}$

[36] Non-linear Methods in HRV Analysis, Procedia Technology, vol.22, 2016, 645-651. https://doi.org/10.1016/j.protcy.2016.01.134

[37] S. Chatterjee. Vision-based rock-type classification of limestone using multi-class support vector machine, Applied Intelligence, 39 (2013), 14-27. https://doi.org/10.1007/s10489012-0391-7

[38] Vapnik, V. N. Statistical Learning Theory. John Wiley and Sons, Inc., New York. 1998.

[39] Yunong Zhang, Shuai Yue, Ke Chen, Chenfu Yi. MATLAB Simulation and Comparison of Zhang Neural Network and Gradient Neural Network for Time-Varying Lyapunov Equation Solving, Advances in Neural Networks, 2008, 117-127. https://doi.org/10.1007/978-3-540$\underline{87732-5 \_14}$

[40] H Francis Song, Guangyu R Yang, and Xiao-Jing Wang. Reward-based training of recurrent neural networks for cognitive and value-based tasks, eLife. 2017; 6: e21492. https://doi.org/10.7554/elife.21492

[41] Andrés Hernández-Serna, Luz Fernanda Jiménez-Segura. Automatic identification of species with neural networks, PeerJ. 2014; 2: e563. https://doi.org/10.7717/ peerj. 563

[42] Bo, Liefeng, Ling Wang and Licheng Jiao. Feature Scaling for Kernel Fisher Discriminant Analysis Using Leave-One-Out Cross Validation. Neural Computation, 18, 2006, 961-978. https://doi.org/10.1162/neco.2006.18.4.961

[43] M. Javanbakht, R. Hejazikenari, M. Ghasemi, Effects of yoga on depression and anxiety of women, complementary therapies in clinical practice, 15 (2) (2009), 102-104. https://doi.org/10.1016/j.ctcp.2009.01.003

[44] I Barnes, PW Crous, MJ Wingfield, BD Wingfield. Multigene phylogenies reveal that red band needle blight of Pinus is caused by two distinct species of Dothistroma, D. septosporum and D. pini. Stud. Mycol. 2004, 50: 551-565. https://edepot.wur.nl/24026. https://doi.org/10.1111/j.1755-0998.2008.02142.x

[45] Travis F, Haaga DA, Hagelin J, Tanner M, Nidich S, Gaylord-King C, Grosswald S, Rainforth M, Schneider RH. Effects of Transcendental Meditation practice on brain functioning and stress reactivity in college students. Int J Psychophysiol. 2009, 71(2):170-176. https://doi.org/10.1016/j.ijpsycho.2008.09.007 
[46] Matousek RH, Dobkin PL, Pruessner J. Cortisol as a marker for improvement in mindfulness-based stress reduction, Complement Ther Clin Pract. 2010; 16(1):13-9. https://doi.org/10.1016/j.ctcp.2009.06.004

[47] Kabat-Zinn J, Massion AO, Kristeller J, Peterson LG, Fletcher KE, Pbert L, Lenderking WR, Santorelli SF. Effectiveness of a meditation-based stress reduction program in the treatment of anxiety disorders. Am J Psychiatry. 1992 Jul; 149(7):936-43. https://doi.org/10.1176/ajp.149.7.936

[48] Kelly, F, Kelly, B. and Ryan, D. Assessment of Psychiatric and Psychological Needs among Help-Seeking Migrants in Dublin, 2008.

[49] MK Moridani, SK Setarehdan, AM Nasrabadi, E Hajinasrollah A novel approach to mortality prediction of ICU cardiovascular patient based on fuzzy logic method; Biomedical Signal Processing and Control; 2018; 45, 160-173.https://doi.org/10.1016/j.bspc.2018. $\underline{05.019}$

[50] Arnsten AF, Rubia K. Neurobiological circuits regulating attention, cognitive control, motivation, and emotion: disruptions in neurodevelopmental psychiatric disorders. J Am Acad Child Adolesc Psychiatry, 2012, 51: 356-367. https://doi.org/10. 1016/j.jaac.2012.01.008

[51] Mohammad Karimi Moridani, Majid Pouladian, A Novel Method to Ischemic Heart Disease Detection Based on Non-Invasive ECG Imaging, Journal of Mechanics in Medicine and Biology; 2019; 19, (3), 1-25. https://doi.org/10.1142/s0219519419500027

\section{Authors}

Mohammad Karimi Moridani received a BS degree in electrical engineering-Electronic in 2006 and his MS and Ph.D. degrees in biomedical engineering-bioelectric in 2008 and 2015, respectively, from Islamic Azad University, Science and Research Branch, Tehran, Iran. Email: karimi.m@iautmu.ac.ir

Now he is an assistant professor in the Biomedical Engineering Department, Faculty of Health, Tehran Medical Sciences, Islamic Azad University, Tehran, Iran. His current research interests are in the field of biomedical signal and image processing, nonlinear time series analysis, and cognitive science. Particular applications include ECG, HRV, and EEG Signal Processing in detection and prediction of diseases, Epileptic Seizure Prediction, pattern recognition and Image Processing for face and beauty recognition, watermarking, etc.

Tina Habikazemi received a BS degree in biomedical engineering-clinical in 2017 from Tehran Medical Science, Islamic Azad University, Tehran, Iran. Her research interests include biomedical signal processing, analysis of nonlinear behavior of ECG signal for cardiac disease detection.

Nahid Khoramabadi received a BS degree in biomedical engineering-clinical in 2017 from Tehran Medical Science, Islamic Azad University, Tehran, Iran. Her research interests include biomedical signal processing, analysis of nonlinear behavior of ECG signal for cardiac disease detection.

Article submitted 2020-03-24. Resubmitted 2020-09-23. Final acceptance 2020-09-24. Final version published as submitted by the authors. 